\title{
QUASI-POSITRONIUM IN POLYETHYLENE TEREPHTHALATE - TEMPERATURE AND MAGNETIC QUENCHING EFFECTS
}

\author{
G. Consolati and F. Quasso \\ Dipartimento di Fisica, Politecnico di Milano \\ Piazza Leonardo da Vinci, 32-20133 Milano, Italy
}

The features of the positron-electron bound system (quasi-positronium, qPs) in the semicrystalline polymer polyethylene terephthalate (mylar) are obtained while studying the dependence of the ortho-qPs lifetime on temperature in the range of $25-105^{\circ} \mathrm{C}$, in the presence as well as in the absence of magnetic fields. In particular, the "anomalous" effect, previously discovered by Bisi et al. at room temperature, is maintained at higher temperature.

PACS numbers: $71.60 .+\mathrm{z}, 78.70 . \mathrm{Bj}$

\section{Introduction}

It is known that in several non-metallic solids an electron positron bound system can be formed, showing characteristics similar to Ps in vacuo (different decays modes for ortho and para sublevels as well as a hyperfine splitting between triplet and singlet substates). For these reasons, we refer to such a bound system as quasi-positronium ( $q P s)$. The formation and the features of $q \mathrm{Ps}$ can be conveniently investigated using the magnetic quenching method. In some cases the magnetic quenching effect was found to be "anomalous", in the sense that it was anomalously high at the field below 5 kGauss. The effect was revealed in some molecular solids [1] and a few crystalline polymers [2, 3]; different models were proposed to explain it $[4,5]$. Up to the present, no anomalous effect was found in amorphous polymers; furthermore, when a comparison between the magnetic quenching in the ordered and disordered phases of the same material was possible, the anomalous effect disappeared when the structural order of the sample was destroyed $[1,3]$. As a consequence, we suggested a correlation between the crystallinity of the sample and the anomalous effect. It can be remarked that in all the investigated cases the specimen under study was maintained at room temperature. With the aim of ascertaining if the magnetic quenching is sensitive or not to temperature, we performed magnetic quenching experiments in polyethylene terephthalate in the temperature range of $25-105^{\circ} \mathrm{C}$. We remember that Mogensen recently proposed a dependence of the magnetic quenching on temperature for some organic compounds [5]. 


\section{Experimental}

The positron source (a few $\mu \mathrm{Ci}$ ) consisted of a droplet of ${ }^{22} \mathrm{Na}$ from a carrier free neutral solution, evaporated between two identical kapton foils $\left(1.08 \mathrm{mg} \mathrm{cm}^{-2}\right)$. The polyethylene terephthalate was purchased by Aldrich (density $1.375 \mathrm{~g} \mathrm{~cm}^{-3}$ ) as pellets, which were afterwards hot stamped in the form of disks of $20 \mathrm{~mm}$ diameter. The source was inserted between two disks in the usual sandwich configuration; the thickness was sufficient to stop all positrons injected into the sample. Care was taken in changing periodically the sample, for avoiding radiation induced damage. The experimental set up was identical to that used in the previous investigations; details can be found in [1]. Here we limit ourselves to mention the resolution of the lifetime spectrometers that resulted 180 ps for the lifetime measurements and 310 ps for the magnetic quenching experiments. The lifetime spectra were analyzed through the Positronfit Extented Program [6]; a correction for positrons annihilated in kapton was taken into account.

\section{Results and discussion}

The time annihilation spectra in polyethy'sne terephthalate were collected at various temperatures in the range of $25-145^{\circ} \mathrm{C}$ and were resolved into three components. Lifetimes and intensities of the longest component $\tau_{3}$, the only one which is appreciably sensitive to temperature, are reported in Table; from magnetic quenching experiments [7], this component is attributed to the decay of ortho-qPs. The values of the corresponding lifetime are plotted in Fig. 1 as a function of temperature; the errors for lifetimes and intensities are $2 \%$ and $3 \%$, respectively. We observe a linear increase with increasing temperature, as well as a marked change in the slope of the straight line around $85^{\circ} \mathrm{C}$. This behaviour can be explained

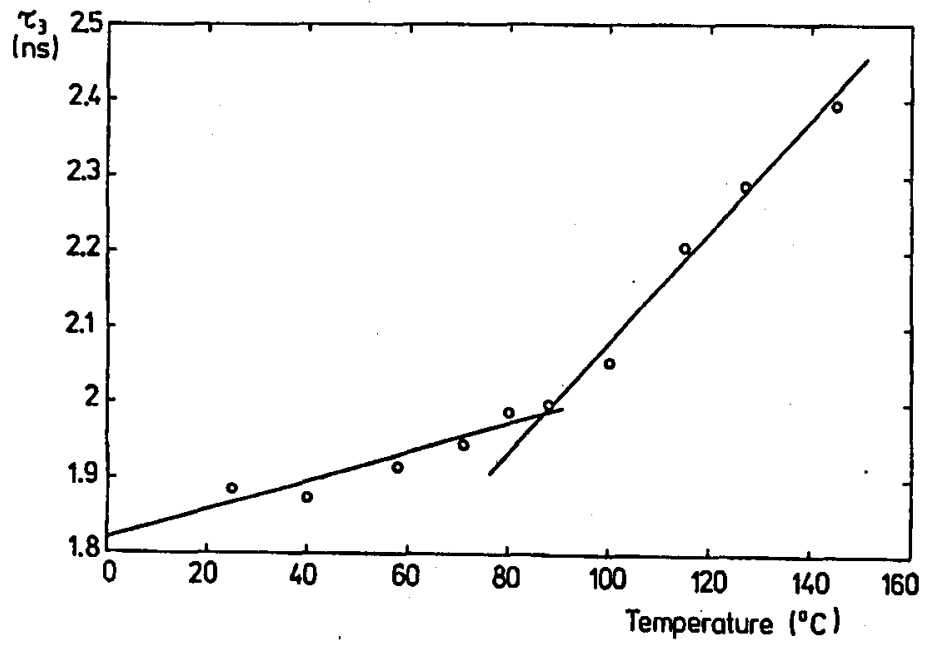

Fig. 1. Ortho-qPs lifetime as a function of temperature. 
in the following way. It is well known that qPs in polymers is formed in the free volume [8]; the ortho-qPs lifetime increases with increasing dimension of the free volume, due to a diminution of the electron density surrounding $\mathrm{qPs}$. Therefore, the linear dependence of the lifetime on temperature reflects the analogous dependence of the free volume. The change in the slope corresponds to the glass transition temperature $\left(T_{\mathrm{g}}\right)$, whose value is in accordance with that obtained through differential scanning calorimetric measurements [9]. For temperature higher than $T_{\mathrm{g}}$, the augmented fraction of the free volume corresponds to an increase of the molecular mobility of the polymeric chain and to correlated main-chain segmental motions; the ortho-qPs lifetime becomes more sensitive to temperature and the linear dependence, after $T_{\mathrm{g}}$, is characterized by a higher slope. Furthermore, the larger is the fraction of the free volume, the higher is the probability of qPs formation; therefore, the intensity of the longest component should increase with temperature, in accordance with the results shown in Table.

TABLE

Temperature dependence of the lifetime $\tau_{3}$ and the intensity $I_{3}$.

\begin{tabular}{c|c|c||c|c|c}
\hline \hline$T\left({ }^{\circ} \mathrm{C}\right)$ & $\tau_{3}(\mathrm{~ns})$ & $I_{3}(\%)$ & $T\left({ }^{\circ} \mathrm{C}\right)$ & $\tau_{3}(\mathrm{~ns})$ & $I_{3}(\%)$ \\
\hline 25 & 1.88 & 16.3 & 88 & 2.00 & 21.5 \\
40 & 1.87 & 17.9 & 100 & 2.05 & 22.3 \\
58 & 1.91 & 19.5 & 115 & 2.21 & 22.1 \\
70 & 1.94 & 20.2 & 127 & 2.29 & 22.5 \\
80 & 1.99 & 20.2 & 145 & 2.40 & 24.1
\end{tabular}

In Fig. 2 the quenching ratio $R$ [1] is reported as a function of magnetic field, taking the sample temperature as a parameter. The quenching ratio $R$ is defined as the ratio of the annihilation events occurring in a prefixed time interval in the presence of the magnetic fields to the same quantity when the field is absent. The time interval is chosen in such a way to include the annihilation events from ortho-qPs as the main contribution.

The $R$ parameter depends on electron density at the positron in $\mathrm{qPs}$, $|\Psi(0)|_{\mathrm{qPs}}^{2} ;$ a relative contact density can be defined as follows:

$\eta=|\Psi(0)|_{\mathrm{qPs}}^{2} /|\Psi(0)|_{\mathrm{Ps}}^{2}$.

In Fig. 2 the continuous line represents the quenching ratio $R$ calculated for an unperturbed ortho-qPs atom $(\eta=1)$ and characterized by the lifetime $\tau=1.88 \mathrm{~ns}$, measured at $25^{\circ} \mathrm{C}$. Varying $\tau$ up to the value corresponding to $105^{\circ} \mathrm{C}$, the calculated $R$ does not change appreciably. On the other hand, the broken curve is calculated with $\eta=0.7$, a typical value for the $\mathrm{qPs}$ contact density in polymers. It is evident that the experimental data are much lower than both the calculated curves; actually, it is impossible to find a value of $\eta$ which fits the data, for each temperature. Therefore, it can be concluded that the anomalous effect in the magnetic quenching is maintained all over the investigated temperatures. Finally, we observe that we limited our measurements between 0 and $5 \mathrm{kGauss,}$ since in this range the anomalous effect is most relevant. 


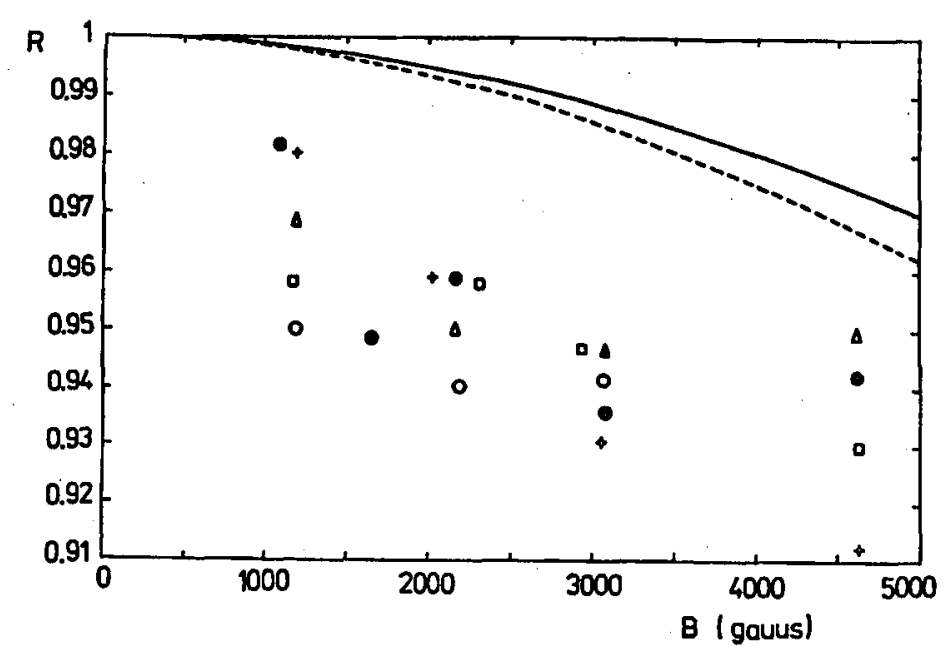

Fig. 2. Quenching ratio $R$ vs. magnetic field at various temperatures $\left(0=25^{\circ} \mathrm{C} ; \Delta=\right.$ $50^{\circ} \mathrm{C} ; \square=70^{\circ} \mathrm{C} ;+=85^{\circ} \mathrm{C} ; \bullet=105^{\circ} \mathrm{C}$ ). Errors for $R$, not indicated for clarity, are typically $1.2 \%$.

\section{Conclusions}

The results presented in the preceding section allow us to make the following observations: The magnetic quenching effect in polyethylene terephthalate remains anomalous when temperature is increased beyond the glass transition temperature $\left(85^{\circ} \mathrm{C}\right)$. After $T_{\mathrm{g}}$, the mean free volume increases and the molecular mobility becomes higher; however, the structural order of the sample, represented by the degree of crystallinity, remains unchanged. Therefore, our hypothesis that the anomalous magnetic quenching effect in solids is correlated to the degree of crystallinity [1] can be maintained on the basis of this experiment.

\section{References}

[1] G. Consolati, F. Quasso, J. Phys., Condens. Matter 2, 3941 (1990).

[2] A. Bisi, G. Consolati, G. Gambarini, L. Zappa, Lett. Nuovo Cimento 40, 176 (1984).

[3] G. Consolati, F. Quasso, J. Phys. C 21, 4143 (1988).

[4] R.S. Brusa, A. Dupasquier, S. Longano, S. Oss, Phys. Rev. B 43, 12715 (1991).

[5] O. Mogensen, Chem. Phys. Lett. 163, 145 (1989).

[6] P. Kirkegaard, M. Eldrup, Comput. Phys. Commun. 7, 401 (1974).

[7] A. Bisi, G. Consolati, G. Gambarini, L. Zappa. Nuovo Cimento D 6, 183 (1985).

[8] Y.C. Jean in: Positron and Positronium Chemistry, Ed. Y.C. Jean, World Scientific, Singapore 1990, p. 1.

[9] M. Pegoraro, private communication. 